\title{
Análisis de sostenibilidad de la política de control de malaria del municipio de El Bagre, Colombia
}

\section{Sustainability Analysis of the Malaria Control Policy in the Municipality of El Bagre, Colombia}

\section{Análise de sustentabilidade da política de controle de malária do município de El Bagre, Colômbia}

Fecha de recepción: 17-02-14 Fecha de aceptación: 17-03-14 Disponible en línea: 01-07-14 doi: 10.11144/Javeriana.rgyps13-27.aspc

Cómo citar este artículo:

Salas-Zapata WA, Ríos-Osorio LA, López-Hernandez LF, Gómez-Arias, RD. Análisis de sostenibilidad de la política de control de malaria del municipio de El Bagre, Colombia. Rev. Gerenc. Polít. Salud. 2014;

13(27): 128-146. http://dx.doi.org/10.11144/Javeriana.rgyps13-27.aspc

\author{
Walter Alfredo Salas-Zapata* \\ Leonardo Alberto Ríos-Osorio \\ Luisa Fernanda López-Hernandez** \\ Rubén Darío Gómez-Arias***
}

Profesor e investigador, miembro del Grupo de Investigación Salud y Sostenibilidad de la Escuela de Microbiología de la Universidad de Antioquia, Calle 67 \# 53-108, bloque 5, oficina 103, Universidad de Antioquia, Ciudad Universitaria, Medellín, Colombia. Correo electrónico: wsalasza@yahoo.es, Tel: +54 (4) 2195489, Fax: +57 (4) 2195486.

** Investigadora, miembro del Grupo de Investigación Salud y Sostenibilidad de la Escuela de Microbiología de la Universidad de Antioquia (Medellín, Colombia).

**** Profesor e investigador, miembro del Grupo de Epidemiología de la Facultad Nacional de Salud Pública de la Universidad de Antioquia (Medellín, Colombia). 


\section{Resumen}

El objetivo de esta investigación fue analizar la sostenibilidad de la política de control de malaria en el municipio de El Bagre durante el año 2011. Se llevó a cabo un estudio de diseño mixto de modelo dominante $C U A L \rightarrow$ cuan, de carácter exploratorio. Se hicieron catorce entrevistas y una revisión documental, para identificar procesos sociales y ecológicos involucrados con la malaria, y también para describir y analizar la política de control de esta enfermedad. Se encontró que la minería, las migraciones, las prácticas y creencias culturales, el conflicto armado y las variaciones climáticas se relacionaban con la dinámica de la enfermedad $\mathrm{y}$, asimismo, que la estructura de la política de control da cuenta de escasa capacidad de los actores para adaptar las acciones de control a estos procesos socioecológicos de la región. La política de control mostró ser efectiva aunque, bajo los supuestos del modelo, es escasamente sostenible.

Palabras clave: desarrollo sostenible; políticas públicas; malaria; modelos teóricos; análisis de sistemas

\section{Abstract}

The objective of this research was to analyze the sustainability of the malaria control policy in the municipality of El Bagre, during 2011. We performed a mixed-design study with a dominant model QUAL $\rightarrow$ quan, of exploratory character. We carried out fourteen interviews and a review of the documentation to identify social and ecological processes involved with malaria, and also to describe and analyze the control policy for this illness. We found that mining, migrations, cultural practices and beliefs, the armed conflict and climate variations are related with the dynamic of the illness. Likewise, we found that the structure of the control policy accounts for the limited capacity of the actors to adapt the control actions to the socio-ecological processes of the region. The control policy showed to be effective, although, under the assumptions of the model, it is barely sustainable.

Keywords: sustainable development; public policies; malaria; theoretical models; systems analysis

\section{Resumo}

O objetivo desta pesquisa foi analisar a sustentabilidade da política de controle de malária no município de El Bagre durante o ano 2011. Efetuou-se estudo de desenho misto de modelo dominante $Q U A L \rightarrow q u a n$, de caráter exploratório. Foram feitas quatorze entrevistas e uma revisão documental, para identificar processos sociais e ecológicos envolvidos na malária, mesmo para descrever e analisar a política de controle desta doença. Encontrou-se que a mineração, migrações, práticas e crenças culturais, o conflito armado e as variações climáticas relacionavam-se com a dinâmica da doença e, do mesmo modo, que a estrutura da política de controle mostra escassa capacidade dos atores para adaptar as ações de controle a esses processos socioecológicos da região. A política de controle mostrou ser efetiva embora, sob os supostos do modelo, escassamente sustentável.

Palavras-chave: desenvolvimento sustentável; políticas públicas; malária; modelos teóricos; análise de sistemas 


\section{Introducción}

Las políticas públicas en salud (PPs) son dispositivos para el control social utilizados por sistemas de actores, que se expresan en sus reglas de acción y definen modos de proceder frente a un problema de salud de interés público. Los propósitos de una política no son siempre explícitos y no benefician a toda la sociedad. Como dispositivos para el control social, las pPs se materializan en actos jurídicos, planes, programas y proyectos, contratos y estrategias ideológicas (1). Usualmente, son influenciadas por un entorno complejo de redes de interacciones que influyen en su desempeño (2) y afectan las posibilidades de cumplir los propósitos que ellas tienen con respecto un problema de interés público.

Las PPS se consideran sostenibles cuando el sistema de actores tiene la capacidad de reconocer las complejas dinámicas socioculturales, económicas y ecológicas involucradas en el problema de salud que se va a intervenir, y de adaptar sus reglas, dispositivos de control y procesos a la complejidad de tales dinámicas, con la intención última de mantener el cumplimiento de los propósitos que estas persiguen (3, p. 115). Así, cuando dicha capacidad es escasa, se corre el riesgo de malgastar recursos públicos, deteriorar la confianza de las comunidades, impedir el mantenimiento de los beneficios en salud, propiciar la reemergencia del problema y poner en peligro los propósitos de la política pública.

En el caso de las políticas de control de malaria (PCM), el problema de interés público es la carga de morbilidad por malaria y la necesidad de mantenerla controlada. Las PCM pueden verse influidas por diferentes dinámicas socioecológicas como la deforestación, las migraciones, las reformas sanitarias, la pobreza, las variaciones climáticas y los 130 efectos de otros proyectos socioambientales (4-7). En ese sentido, una PCM es sostenible cuando el sistema de actores tiene la capacidad de reajustar sus reglas y dispositivos de control para hacer frente a los procesos o dinámicas socioecológicas relacionadas con la malaria y mantener el cumplimiento de los objetivos de la política en términos de control de la enfermedad (3, p. 126).

En Colombia, el Bajo Cauca antioqueño ha sido una de las regiones más afectadas por este problema (5). En esta región se encuentra el municipio de El Bagre, una localidad de 47875 habitantes (8) que se extiende en 1653 $\mathrm{km}^{2}$ en una zona de bosque húmedo tropical, a una altura de $50 \mathrm{~m}$, y con una temperatura media anual de 26,8ㅇ (9). Estas características son propicias para la transmisión de la enfermedad, y ello se ve reflejado en las altas incidencias que reporta el municipio (10). Dado que las características de esta localidad hacen que el control de la malaria sea aún más desafiante para cualquier política pública, este estudio se propuso analizar la sostenibilidad de la Рсм que se estaba llevando a cabo durante el año 2011.

\section{Métodos}

\section{El modelo teórico utilizado y el tipo de estudio}

Se utilizó un modelo teórico desarrollado por Salas-Zapata (3) para analizar la sostenibilidad de las pps. Este modelo entiende sostenibilidad como la resiliencia socioecológica de los sistemas; en este caso el sistema objeto de análisis es la PCM en El Bagre. El modelo orientó tanto la recolección como el análisis de información (figura 1).

El estudio se llevó a cabo siguiendo un diseño mixto de modelo dominante $C U A L \rightarrow$ cuank, de carácter exploratorio (figura 2). Para la recolección de la información correspondiente a los componentes A y B, de forma 
paralela se hizo una revisión documental y se hicieron entrevistas. A partir de estos métodos se identificaron dinámicas socioecológicas, y con base en datos estadísticos se analizó la relación entre los procesos sociales y ecológicos hallados y el comportamiento de la malaria. Para analizar estas relaciones se utilizaron correlaciones de Pearson y correlaciones cruzadas (componente A). A partir de estos métodos, también se describió la PCM (componente B) y se hizo triangulación con los informantes. Luego se utilizaron estos resultados para analizar el componente $\mathrm{C}$.

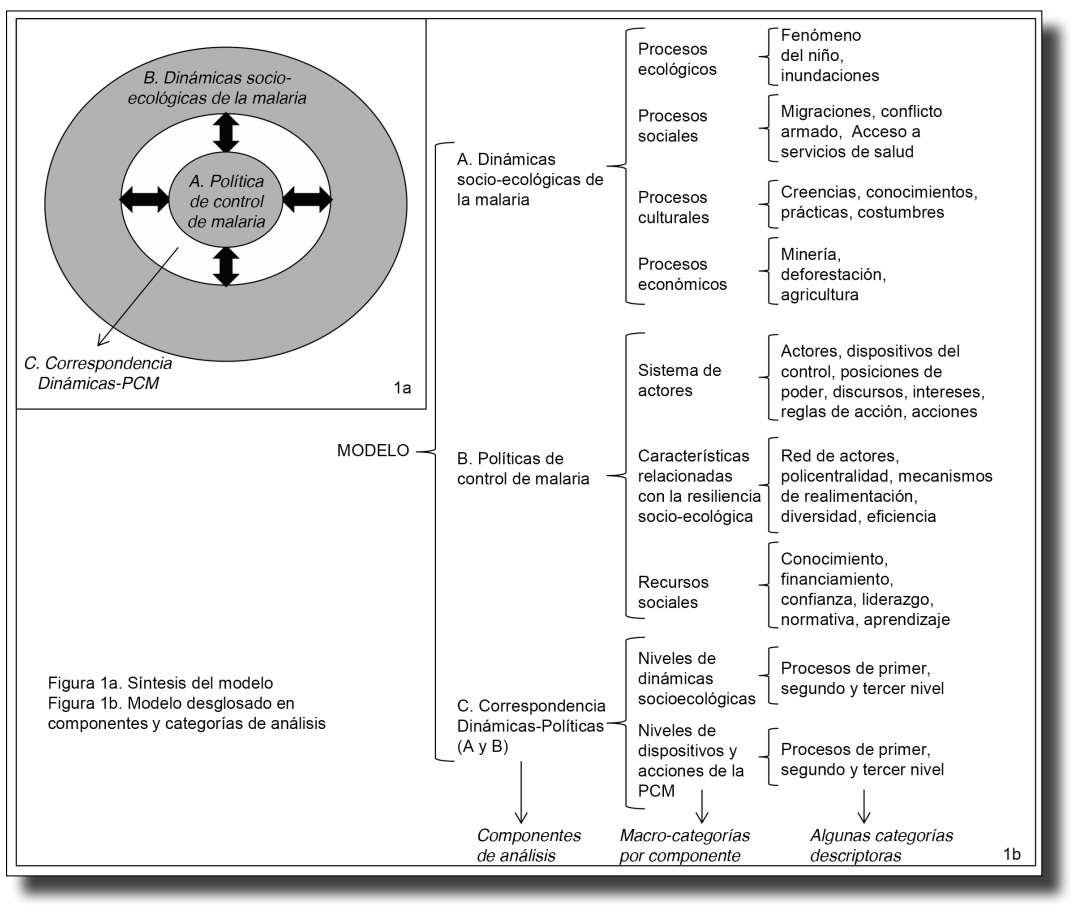

Figura 1. Modelo de análisis de SOSTENibilidad de PCM

El modelo se fundamenta en el concepto de resiliencia socioecológica de los sistemas, que es la capacidad que tiene un sistema de reorganizar adaptativamente el arreglo de interacciones socioecológicas en respuesta a perturbaciones para mantener sus atributos esenciales. En este caso, las perturbaciones son los procesos sociales y ecológicos que afectan el comportamiento de la malaria y su control en la localidad; el sistema es la pcm propiamente dicha, y se asume el control de la malaria como un atributo esencial. De ese modo, para analizar la sostenibilidad de una pcm, el modelo sugiere analizar tres componentes de las pcm: las dinámicas socioecológicas de la malaria y su control (componente A); la política de control de malaria (componente B); y la correspondencia que existe entre las pcm y las dinámicas de la malaria (componente C). Con respecto a este último, se asume que en la medida en que haya más capacidad adaptativa, las reglas de acción y dispositivos de control serán coherentes con los procesos sociales y ecológicos relacionados con la malaria. Para el análisis de cada componente es necesario dar cuenta de unas macrocategorías. A su vez, estas pueden ser estudiadas mediante la descripción de algunas posibles categorías emergentes. Lo anterior significa, por tanto, que no necesariamente surgirán todas las categorías señaladas en la figura 1.

Fuente: basado en Salas-Zapata (3) 
Walter Alfredo Salas-Zapata • Leonardo Alberto Rios-Osorio • Luisa Fernanda López-Hernandez • Rubén Darío Gómez-Arias

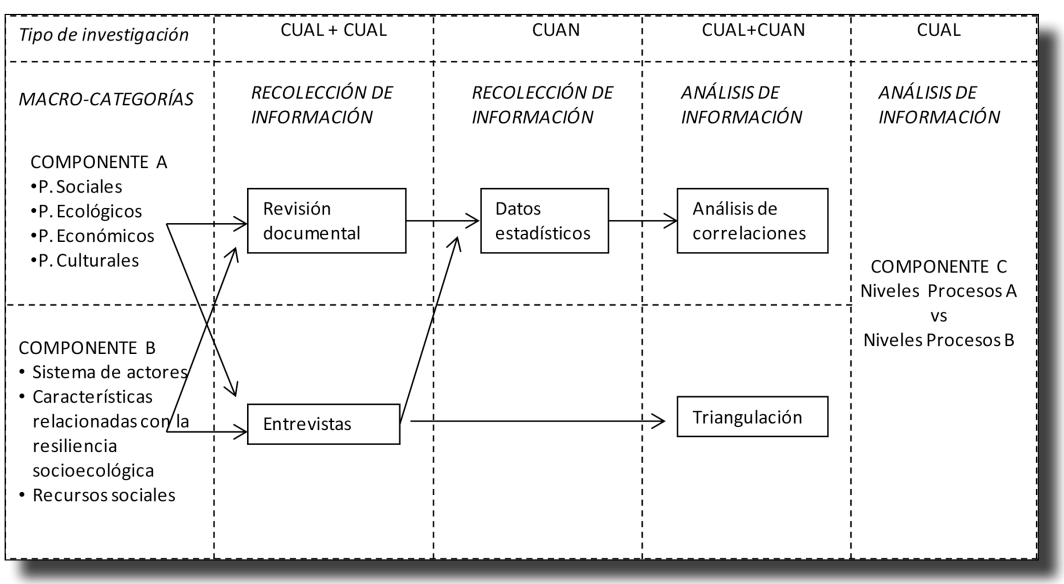

Figura 2. MÉtodos

Fuente: elaboración propia

\section{Recolección y análisis de información}

Con respecto a las entrevistas, la selección de los informantes clave se hizo utilizando como criterio el "sujeto que desempeñara algún rol dentro del control de la malaria en El Bagre". El muestreo de los sujetos se hizo en bola de nieve. Se entrevistó a catorce sujetos, entre los que se encontraban funcionarios de la Secretaría Seccional de Salud de Antioquia, funcionarios de la Secretaría de Salud de El Bagre, de la Organización Panamericana de la Salud (ops), bacteriólogos, microscopistas y personas contratadas a través de la Fundación Universidad de Antioquia que trabajan en acciones de control. A cada participante se le hizo una entrevista abierta con preguntas orientadas hacia tres ejes: 1) su participación y vínculo con las actividades y procesos relacionados con el control de la malaria en el municipio, 2) su conocimiento y percepción de la estructura, los actores y el funcionamiento de la PCM, y 3) su conocimiento y percepciones sobre el comportamiento de la malaria en la zona y los aspectos sociales y ecológicos del municipio que afectan su control. Las preguntas 132 indagaron por una perspectiva temporal de los últimos veinte años.
Para la revisión documental se tuvieron en cuenta artículos de revistas científicas, manuscritos, libros, reportes de prensa e informes de agencias gubernamentales y no gubernamentales, que se refirieran al tema de la malaria y su control, específicamente en el municipio de El Bagre.

Los testimonios de las entrevistas fueron grabados y transcritos en el programa Microsoft Word. Los textos fueron analizados según las macrocategorías de los componentes A y B. Una vez agrupados por macrocategorías, se identificaron tendencias en los discursos para revelar categorías emergentes. De igual manera, los documentos producto de la revisión fueron analizados según las macrocategorías en mención.

A partir de las categorías que emergieron de las entrevistas y de la revisión documental, se identificaron procesos sociales y ecológicos que los participantes percibían que estaban relacionados con la malaria (dinámicas socioecológicas relacionadas con la malaria y su control). Para aumentar la validez de los hallazgos referidos a estas relaciones, estas fueron analizadas estadísticamente mediante análisis de correlaciones y de correlaciones cruzadas, utilizando el software R, de libre circulación. 
El sistema de actores, las características relacionadas con la resiliencia socioecológica y los recursos sociales de los que depende la PCM, se describieron a partir de las categorías reveladas por las entrevistas y la revisión documental. Se elaboró un documento con la descripción de la PCM y para validar los hallazgos se hizo una triangulación con los entrevistados que tenían más conocimiento sobre la PCM y la malaria en la región.

Una vez reunidos los hallazgos de los componentes A y B del modelo, se procedió a analizar el componente C. Esto se hizo clasificando las dinámicas sociales y ecológicas de la malaria en tres niveles, así: en el primer nivel se ubican los procesos sociales y ecológicos que afectan directamente la posibilidad de ocurrencia de casos de malaria; en el segundo nivel, aquellos que afectan directamente a los de primer nivel; y así, sucesivamente, los de tercer nivel. Las reglas y dispositivos de control se organizaron siguiendo la misma lógica, para analizar la coherencia entre las reglas y dispositivos de control de los actores y los procesos sociales y ecológicos relacionados con la malaria.

\section{Resultados}

Componente A: dinámicas socioecológicas de El Bagre relacionadas con la malaria

La minería, las migraciones, las prácticas y creencias culturales, el conflicto armado y las variaciones climáticas se identificaron como procesos relacionados con la dinámica de la enfermedad en la localidad y con los intereses e interacciones de los actores.

Malaria y minería. Los testimonios de los entrevistados sugieren que el comportamiento de la malaria en el municipio está relacionado con la minería: "la malaria se ve más en los mineros o en las personas que viven cerca de las minas" (E3). En efecto, cuando empezó el auge minero, se dio un incremento de los casos: "hace unos años había mucha malaria también, [...] pero no tan alarmante como ahora [...] cuando surgió el apogeo de la minería surgió la malaria y los picos fueron demasiado grandes" (E1). Los entrevistados consideran que durante la explotación minera, las retroexcavadoras hacen socavones, dejan huecos en los caminos y forman charcos cubiertos por vegetación y materia orgánica que se llenan de agua y se convierten en criaderos de mosquitos.

Los registros oficiales de malaria entre enero y diciembre del 2011 muestran que cerca del $30 \%$ de los 7705 casos registrados correspondían a población dedicada a actividades relacionadas con la minería (figura 3). Según los entrevistados, los mineros más afectados son aquellos dedicados a la pequeña minería, a la minería informal y a la ilegal. De hecho, esta enfermedad es muy escasa entre los trabajadores de empresas como Mineros S. A., debido a que la actividad mecanizada y la protección que estos utilizan los protegen de la exposición al vector [E8].

Asumiendo que el incentivo de los mineros para internarse en la selva es el precio del oro, se analizó la relación entre el precio internacional de la onza de este mineral y el número de casos de malaria registrados entre el año 2000 y el 2010 (figura 4), y se encontró (figura 4) una correlación positiva entre ambas variables (coeficiente de Pearson $=0,73$ ). El comportamiento de la malaria se aleja en dos ocasiones del comportamiento del precio del oro (años 2007 y 2010). Estas reducciones pueden ser atribuibles a cambios en las directrices y acciones de control.

\section{Malaria y migraciones, prácticas y creencias.} A partir de las entrevistas se infiere que la migración poblacional, relacionada con la actividad minera en la región, limita también 


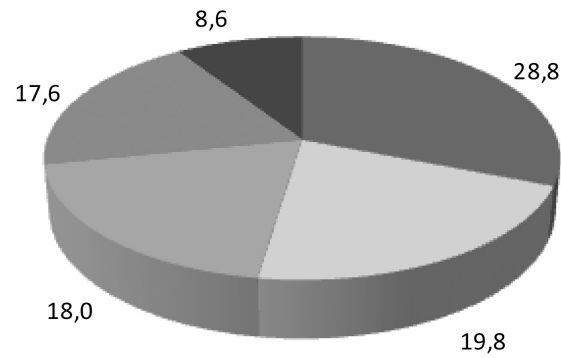

- Minería

Menor de edad

Hogar y madres comunitarias

Estudiante

- Obreros de carga

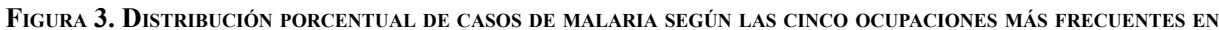
El Bagre (ANTIOQuia) en el año 2011

Estas cinco ocupaciones representan el 92,8\% del total de casos reportados. Por esa razón, los porcentajes no suman $100 \%$, sino 92,8\%. La enfermedad se distribuye ampliamente en la población.

Fuente: base de datos suministrada por la Secretaría de Salud de El Bagre

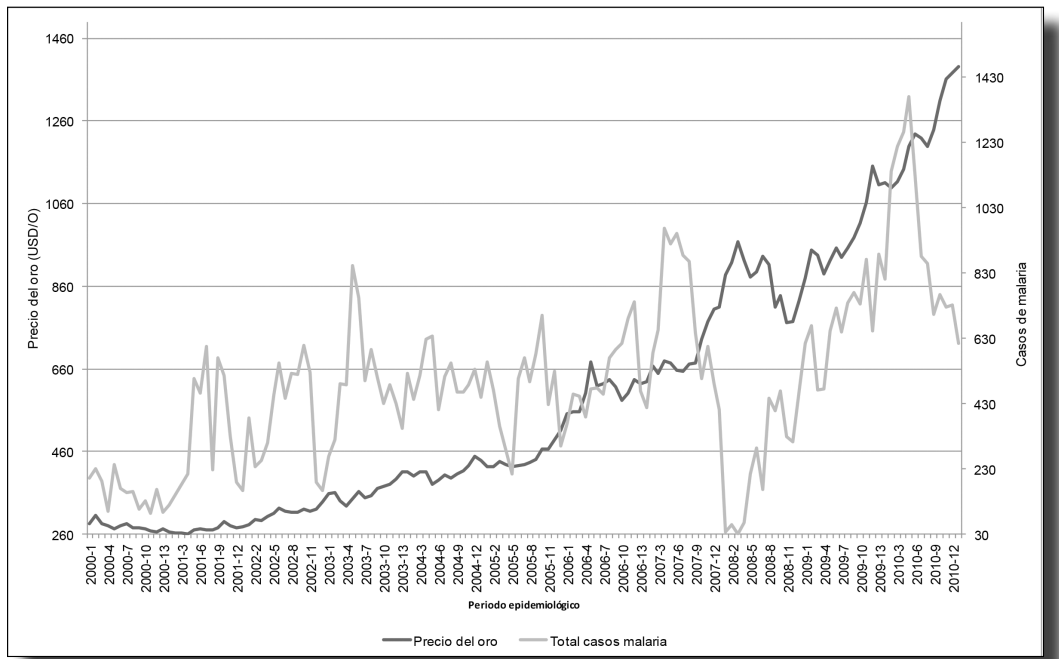

Figura 4. Dinámica del PRecio del oro y de los Casos de malaria

Fuente: los datos de malaria fueron suministrados por la Secretaría Seccional de Salud de Antioquia y los del precio del oro fueron obtenidos del portal en Internet de Kitco (http://charts.kitco.com/KitcoCharts/).

el control de la malaria: "En la mayoría de comunidades existe la minería [...] en estas zonas es más difícil la participación, (los mineros) llegan ahí y se establecen un tiempo y luego se internan otro tiempo allá, pero 'allá' no hay sentido de pertenencia” (E5). La población minera "es muy flotante, llegan tres o cuatro meses y se van" (E5), y son poco participativas. Una frase que resume la idiosincrasia de los mineros (informales, pequeños, ilegales) puede ser "yo vine a sacarme una platica en la mina y cuando la tenga me voy" (E5). En este tipo de población es difícil controlar la malaria, porque "le entregas un toldillo a una persona y mañana esa persona ya no está" (E4). La alta movilidad de la po- 
blación hace más difícil controlar la malaria y puede relacionarse con la persistencia de la enfermedad.

Alrededor de las zonas de explotación puede haber caseríos, unos fijos y otros transitorios que son construidos y habitados por los mineros mientras la mina está activa: "Hay caseríos que no están dedicados a la minería pero indirectamente sí viven de ello, los que tienen restaurantes, o venden cerveza [...] hay caseríos de 30, 40, 50 casas. Como están tan asociados a las minas hay caseríos que sí son fijos de 40, 50 casas, pero hay otros que dependen [...], si están explotando la mina hay, (y) entonces arman su cambuche de plástico mientras se explota la mina y al otro mes o seis meses ya no están" (E9-10). Esta transitoriedad también impide que haya continuidad en las acciones educativas y, por eso, también se hace difícil lograr aprendizajes y cambios culturales en la población que favorezcan el control.

Algunas creencias influyen en la dinámica de la malaria. Según los entrevistados, la gente cree que la malaria se transmite al "bañarse con agua del río" (E3), o por tomar agua sucia o cruda, y no asocian la enfermedad con la exposición al mosquito ni con la hora en la que se bañan en el río. Esta población, que en su mayoría trabaja en zonas rurales, suele bañarse entre cuatro y cinco de la tarde, cuando comúnmente pica el mosquito. Ello supone una creencia que lleva a las personas a exponerse a la enfermedad en las zonas y horas de mayor riesgo. La relación entre la malaria y las prácticas y creencias de las comunidades en la localidad ya ha sido documentada (11), se encontró que la participación de estas comunidades en acciones de control era también escasa, al parecer debido a su carácter flotante. No obstante, las costumbres y el comportamiento frente a la malaria pueden ser muy diferentes de unas comunidades a otras. Por ejemplo, las comunidades indígenas han dado una buena respuesta a las intervenciones, debido a que son comunidades organizadas y capacitadas y tienen líderes y gobernadores (E1).

Malaria y conflicto armado. Los testimonios recogidos sugieren también que el deterioro de la situación de orden público a causa del conflicto armado afecta en diferentes formas la posibilidad de realizar acciones de control de la malaria. Por ejemplo, resulta difícil seguir los casos de malaria de miembros de grupos armados ilegales: “acá existe un problema con los documentos de identidad, ya que la gente se inventa los números de cedula; debido a que existen muchos grupos al margen de la ley, estas personas se cambian el nombre, el número de la cédula, lo que causa una desinformación y confusión en las bases de datos" (E4). Por otra parte, cuando hay riesgo de enfrentamientos armados entre los grupos ilegales y las fuerzas del Estado, el acceso a las poblaciones para adelantar labores de control de la enfermedad se limita. Un criterio de selección de las localidades para hacer la estrategia Combi ${ }^{1}$ es "que no haya problemas de orden público, [...] porque ha tocado cambiar [...] en este año se tocó cambiar localidades por eso" (E5).

Para evaluar la relación entre la enfermedad y el conflicto armado, se comparó el número de casos de malaria registrados en El Bagre y en el Bajo Cauca con el número de enfrentamientos armados ocurridos entre el 2000 y el 2010; se encontró correlación positiva (test de Pearson 0,79 y 0,75 , respectivamente) (figura 5).

Malaria y variaciones climáticas. Los testimonios de los entrevistados no relacionaron la malaria con el clima, aunque la literatura

1 Estrategia de trabajo comunitario Combi, de su nombre en inglés: Communication for Behavioral Impact. En castellano: Comunicación para el impacto conductual. 


\section{Walter Alfredo Salas-Zapata • Leonardo Alberto Rios-Osorio • Luisa Fernanda}

López-Hernandez • Rubén Darío Gómez-Arias

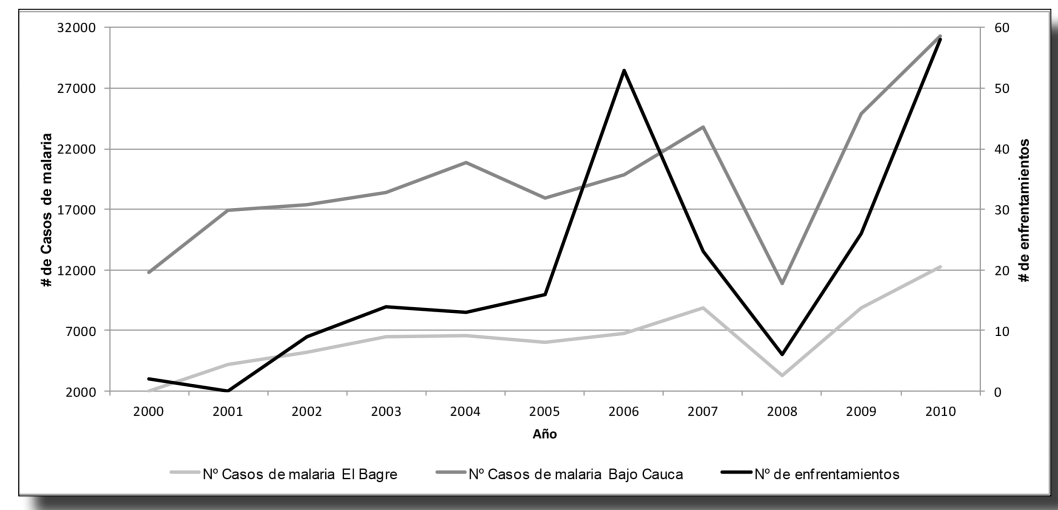

Figura 5. Tendencia del número de casos de malaria en El Bagre y El Bajo Cauca según número de enfrentamientos anuales en El Bajo Cauca, 2000-2010

Los datos oficiales de población registran una reducción drástica del $20 \%$ de la población de El Bagre entre el 2004 y el 2005. Este cambio puede atribuirse a que los datos del 2004 son producto de las proyecciones del censo de 1993 mientras que las del 2005 se obtuvieron en el censo del 2005. No se utilizaron tasas de malaria porque, en este caso, tendría un efecto más perturbador sobre la percepción de la realidad que la utilización del número de casos.

Fuente: Los datos de los enfrentamientos fueron suministrados por la Fundación Ideas para la Paz. Enfrentamientos vs. malaria Bagre: p-valor $=0,004$, confianza del 95\%. Enfrentamientos vs. malaria Bajo Cauca: p-valor $=0,007$, confianza del $95 \%$

ya ha destacado la relación entre la malaria y las variaciones climáticas en El Bagre (12), así como la aparición de picos de malaria después de elevaciones de temperatura generados por el fenómeno del Niño (7). A este respecto, se hizo un análisis de correlaciones cruzadas entre los registros de temperatura, humedad relativa, precipitación y casos de malaria en el periodo 2000-2010 (tabla 1), y se halló una relación débil entre las variables climáticas y el rezago en los casos de mala- ria. La mayor correlación se observó entre la temperatura y el rezago de dos periodos epidemiológicos $(0,336)$.

\section{La política de control de malaria en El Bagre}

El sistema de actores. En términos generales, se puede considerar que las acciones de control de malaria son de tres tipos. El primero corresponde a las acciones de

TABLA 1. Matriz de Correlaciones CruZadas de variables Climáticas vs. Malaria

\begin{tabular}{|l|l|l|l|}
\hline $\begin{array}{l}\text { Rezago (periodo epide- } \\
\text { miológico) }\end{array}$ & $\begin{array}{l}\text { Temperatura vs. casos } \\
\text { malaria }\end{array}$ & $\begin{array}{l}\text { Precipitación vs. casos } \\
\text { malaria }\end{array}$ & $\begin{array}{l}\text { Humedad relativa vs. } \\
\text { casos malaria }\end{array}$ \\
\hline 1 & 0,319 & 0,083 & 0,213 \\
\hline 2 & 0,336 & $-0,004$ & 0,161 \\
\hline 3 & 0,301 & $-0,082$ & 0,196 \\
\hline 4 & 0,222 & $-0,132$ & 0,217 \\
\hline 5 & 0,173 & $-0,126$ & 0,248 \\
\hline
\end{tabular}

Los datos climáticos fueron suministrados por el Ideam y corresponden a la estación de Caseri. Con el fin de obtener periodos epidemiológicos completos en todas las variables, los años que se encontraron con información incompleta fueron eliminados del análisis. Lo anterior llevó a eliminar de la base de datos la información que se tiene para los periodos epidemiológicos comprendidos entre 2007-1 y 2007-7 y 2009-6 y 2009-13.

Fuente: elaboración propia 
diagnóstico y tratamiento que son llevadas a cabo por los microscopistas en las veredas, en los laboratorios clínicos y en los centros y puestos de salud. El segundo corresponde a las acciones de investigación del riesgo y el control de vectores, que son desarrolladas por la Secretaría Departamental de Salud (SDS) o por la Universidad CES y el personal contratado por ellos. El tercero corresponde a las acciones de educación y prevención con las comunidades, llevadas a cabo por los agentes Combi y otros profesionales de las ciencias sociales que hayan sido designados por la SDS.

El personal encargado de las acciones de educación y prevención a nivel comunitario, así como aquellos encargados del control de vectores e investigación de riesgo, entregan informes directamente a la SDS. Por su parte, los microscopistas reportan los casos positivos y solicitan los respectivos medicamentos a la Secretaría de Salud Municipal (SSM). Esta acopia la información y la transfiere a la SDS, la que a su vez se encarga de planear, coordinar y contratar las actividades de control que se han de desarrollar en el municipio. Lo anterior significa que en el control de la malaria, la sDs es un actor central porque recibe la información de todas las acciones que se llevan a cabo en el municipio, puede ejecutar recursos, y decide el modo de proceder frente a la enfermedad. La sDs actúa como canal de información y reporte de los microscopistas y apoya la planeación de actividades.

Durante la última década, este ha sido el funcionamiento del control de la malaria en el municipio. Desde el 2010 empezó a implementarse el "Proyecto Malaria Colombia", más conocido entre los entrevistados como

2 El proyecto se llama: Uso de la inteligencia epidemiológica con participación social para fortalecer el programa de manejo, el acceso al diagnóstico y al tratamiento, y la ejecución de intervenciones efectivas para la prevención y el control de malaria. el "Proyecto del Fondo Global" (PFG), y un nuevo actor empezó a incursionar en el control de la malaria. La figura 6 describe el sistema de actores al momento del estudio.

Cuando iniciaron las acciones promovidas por el Fondo Global se generaron conflictos entre el PFG y el programa regular de control de malaria de la sDs que afectaron el desarrollo de las acciones de control. Esto se debió a varias razones. La configuración de una aparente incursión paralela de equipos de trabajo diferentes es una de ellas. Los entrevistados señalan que inicialmente estos "eran cosas totalmente desligadas" (E9-10); "el proyecto estaba andando solo sin mucho apoyo (de la SDS), por el tema de información" (E5); "había dos coordinaciones paralelas" (E12); "cuando llegó el proyecto del Fondo Global llegó un despelote" (E910). Los funcionarios del programa regular de la sDs sintieron que el PFG llegó desconociendo o descalificando su trabajo. Algunos entrevistados manifestaron que el proyecto "(entró) diciendo que el personal viejo no ha hecho nada" (E12). "Ellos decían que en Antioquia se entregaban toldillos de larga duración pero que nosotros no sabíamos cómo hacerlo" (E9-10). Asimismo, mencionaron que el PFG, por ejemplo, "enfocó el problema de malaria en Antioquia como si fuera el mismo problema que hay en el Chocó, en Córdoba, en Cauca y en Valle, entonces [...] [asumieron] que ellos venían a hacer la red de microscopía [...] [cuando esta] ya existía como tal” (E12).

Por otra parte, a diferencia de otros departamentos de Colombia, la red de microscopía estaba compuesta en Antioquia solamente por microscopistas voluntarios. Según los entrevistados, cuando llegó el PFG "ellos llegan a pagarle al microscopista [...] (y) se desestabiliza lo que nosotros tenemos". Por ejemplo, “(un) curso (de capacitación) lo estábamos haciendo nosotros y llegaron 


\section{Walter Alfredo Salas-Zapata • Leonardo Alberto Rios-Osorio • Luisa Fernanda}

López-Hernandez • Rubén Darío Gómez-Arias

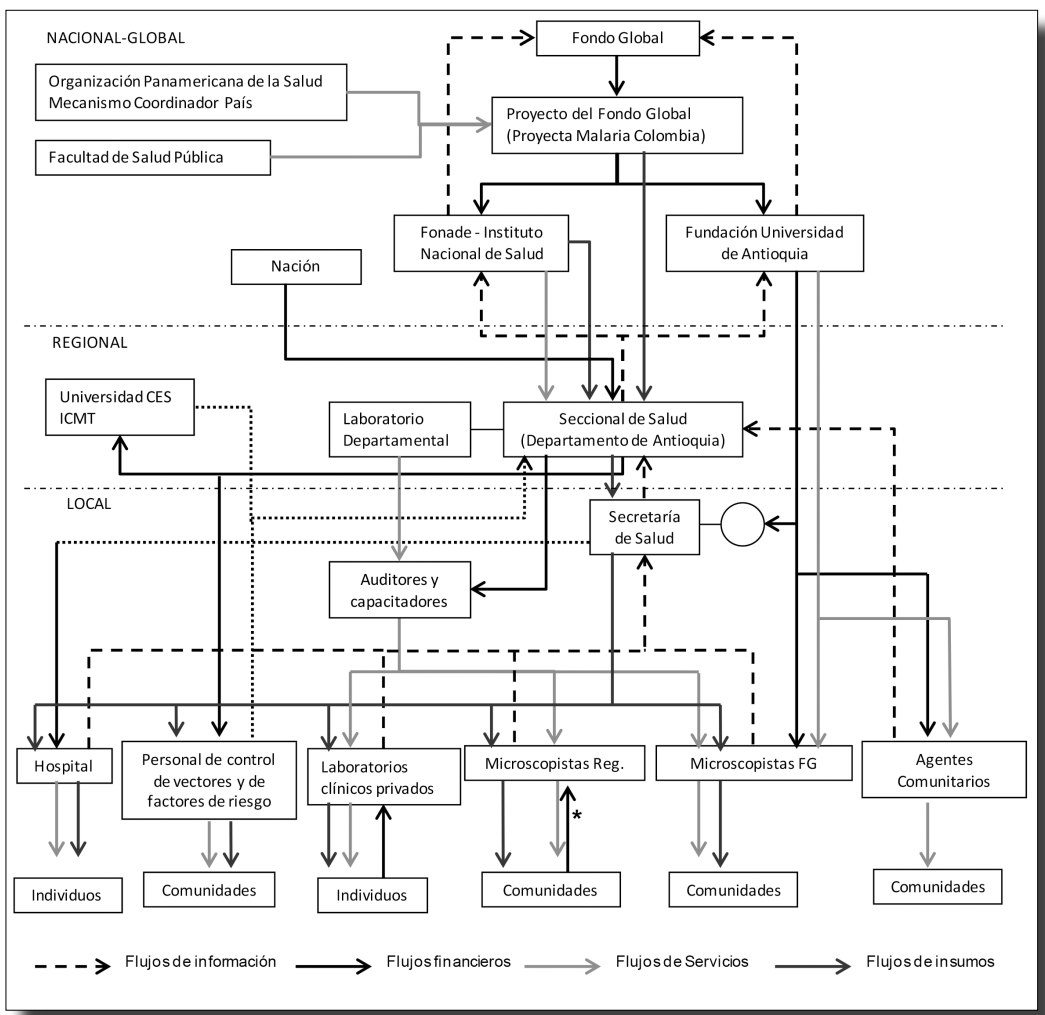

\section{Figura 6. Sistema de aCtores}

Las relaciones entre los actores se materializan en flujos de información, flujos de recursos financieros, flujos de servicios que puede prestar un actor a otro y flujos de insumos. La sds es el actor que más flujos concentra.

Fuente: elaboración propia

en este punto a ofrecerles trabajo entonces, los (microscopistas) que nos quedaron en el trabajo [...] iah problema! [...] son los que cobran 10000 o 15000 [pesos], ${ }^{3}$ los que va a ver que cerrarles el puesto de microscopia (por cobrar algo que se supone es gratis). Muchos ya desertaron pues se salieron, [...] a los que les pagan entonces los han cogido [contratado]" (E9-10). Los microscopistas voluntarios revaluaron su compromiso: "Cuando yo ya empiezo a hacer [las reuniones] [...] ellos ya no me van ¿por qué? Porque es que a ellos les pagan todo [...] les pagan los pasajes (para ir a las capacitaciones)" (E12). Quienes están a favor del pago a los

3 La actividad del microscopista es voluntaria, no debe cobrar por ello. microscopistas aducen que la labor de estos se ha convertido en un trabajo, pues ya no realizan tres o cuatro lecturas al día, sino muchas más y, en muchos casos, ellos han tenido que cubrir los gastos de transporte y otros insumos. Además, señalan que el pago reduciría el incentivo para vender el medicamento, que también es gratis.

El conflicto entre ambos programas influyó notablemente en la dinámica posterior de las políticas de control. El coordinador para Antioquia del PFG renunció y desde ese momento las actividades derivadas del proyecto pasaron a ser coordinadas directamente por el coordinador del programa de control de la SDS. El flujo de información entre los funcionarios de uno y otro lado mejoró, la 
coordinación de las actividades permitió llegar a más veredas y el control de la malaria se fortaleció en El Bagre.

\section{Características relacionadas con la resiliencia} socioecológica. El sistema de actores involucrados en el control de la malaria tiende a ser centralizado, tiene escasa diversidad, y relativamente pocos mecanismos de retroalimentación. La figura 6 ilustra la falta de policentralidad. El actor central es la SDS, la cual ejerce control sobre todos los flujos o procesos que relacionan a los actores. No se observaron agrupaciones o núcleos de actores en el nivel local.

La tabla 2 describe el sistema de reglas de los actores - arreglo institucional — que da lugar a la PCM. En esta se puede observar escasa diversidad institucional, en tanto existen pocas reglas (en negrita) complementarias que permiten la sustitución de actores en el cumplimiento de un rol o de procesos alternativos para lograr un mismo fin. Esto significa que en raras ocasiones los actores pueden sustituirse entre sí, o que al agotarse una vía de acción para controlar la malaria es difícil encontrar otras opciones. De igual modo, se pueden observar escasos mecanismos de retroalimentación (señalados con un asterisco). Un mecanismo encontrado es el "reporte y notificación de casos", así como el de "auditorías a los microscopistas en el terreno", aunque este último apenas comienza. No hay reglas que lleven a los actores a observar el comportamiento de la minería, el clima, el conflicto y las migraciones, que son en últimas los factores responsables de la persistencia de la enfermedad. En letra cursiva y doble asterisco se encuentra reglas en uso que son contradictorias.

La eficiencia y los recursos sociales de los que depende la política de control. El PFG inicio en el 2010, y desde el 2011 se observa una tendencia hacia la reducción de casos registrados. Considerando que en este periodo aumentaron los microscopistas, los datos sugieren que las acciones de control mejoraron su efectividad. Entre los recursos sociales que pudieron influir en esta tendencia se encontraron:

Financiamiento y personal. La participación del proyecto del Fondo Global supuso un incremento en los recursos financieros, en el personal y en los insumos en general para las acciones que se desarrollan. Su finalización en el 2015 supondría una reducción en este tipo de recursos y ello afectaría las posibilidades de mantener la efectividad que se ha visto hasta entonces.

Voluntad. Probablemente fue el mayor soporte de la red de microscopistas, hasta el 2010 cuando se empezó a pagar a un sector de ellos.

Liderazgo. Es una cualidad necesaria para que los agentes comunitarios (líderes comunitarios de apoyo) puedan potenciar el desarrollo de los Combis y de las actividades de control de vectores en las comunidades. Con su contratación por parte del PFG es posible que el liderazgo endógeno de los agentes comunitarios, basado en el compromiso voluntario, se vea afectado en el futuro.

Memoria y aprendizaje colectivo. En una población flotante como la de El Bagre, las oportunidades de generar aprendizajes colectivos que sean culturalmente transmitidos en la comunidad son escasas. La "memoria colectiva" sobre el control de la malaria pudo detectarse en algunos entrevistados de la sDs, pero no en aquellos actores cuya relación con el programa es contractual o transitoria.

Regulaciones. Aunque la participación local en el desarrollo de las actividades de control es posible a la luz de normas vigentes, como la Resolución 412/2000 y los protocolos de vigilancia epidemiológica que la señalan como necesaria, no se encontró evidencia de toma de decisiones con participación comunitaria. 
TABLA 2. Reglas EN USO QUe ESTRUCTURAN LA POLÍtica de CONTROL DE MALARIA

Reglas del ámbito global-nacional

La Secretaría Seccional de Salud debe ejecutar las acciones de inspección, vigilancia y control de la malaria en El Bagre.

El proyecto del Fondo Global debe reducir la mortalidad y morbilidad por malaria, mediante el fortalecimiento de los programas departamentales.

La Secretaría Seccional de Salud debe coordinar las acciones del proyecto del Fondo Global.

La Fundación Universidad de Antioquia (UdeA) y el Instituto Nacional de Salud (INS) deben administrar financieramente el proyecto.

La ors puede asesorar el proyecto del Fondo Global.

La nación debe transferir recursos al departamento para las acciones de inspección, vigilancia y control de la malaria.

Reglas del ámbito regional

La Seccional de Salud debe entregar informes a la Fundación UdeA y al INS.

La Seccional de Salud debe distribuir y entregar los toldillos en las comunidades.

La Seccional de Salud debe hacer la fumigación en las zonas para control entomológico.

** La Seccional de Salud debe capacitar a los microscopistas.

La Seccional de Salud debe encargarse de hacer gota gruesa en las zonas rurales de El Bagre.

La Seccional de Salud debe entregar los medicamentos a las secretarías de salud.

El proyecto del Fondo Global debe implementar Combi.

La Seccional de Salud debe implementar Combi.

El proyecto del Fondo Global debe comprar toldillos y entregarlos a la Seccional.

** El proyecto del Fondo Global debe capacitar a los microscopistas.

La Seccional de Salud debe notificar los casos de malaria al Instituto Nacional de Salud.

La Facultad de Salud Pública debe asesorar la implementación de la Estrategia Combi.

\section{Reglas del ámbito local}

El laboratorio departamental debe hacer control de calidad a las lecturas de los microscopistas.

Los auditores deben hacer control de calidad a las lecturas de los microscopistas.

La Secretaría de Salud debe entregar los medicamentos a los microscopistas y puestos de diagnóstico.

* La Secretaría de Salud debe notificar los casos de malaria a la Seccional.

La Fundación Universidad de Antioquia puede contratar personal para apoyar, la notificación, la implementación de los Combi, y hacer gota gruesa.

Reglas local-operativo

La lectura de gota gruesa es gratis para todas las personas.

La lectura de gota gruesa puede ser cobrada por los laboratorios privados.

Los microscopistas pueden recibir compensación económica voluntaria por los gastos y trámites.

** La labor de microscopista es voluntaria y no recibe salario.

** La labor de microscopista es contratada y debe recibir un salario.

Los microscopistas deben reportar los casos positivos a la Secretaría de Salud.

Los microscopistas deben entregar el medicamento a las personas con resultados positivos.

* Los auditores deben supervisar la labor de los microscopistas.

El medicamento para tratar la malaria es gratis para las personas.

Si un microscopista cobra por hacer la gota gruesa y entregar el medicamento, su puesto debe cerrarse.

Un profesional del área social debe implementar la estrategia Combi en las comunidades.

El agente comunitario debe promover la organización comunitaria para fortalecer los Combi.

$\mathrm{El}$ agente comunitario debe ser contratado por el proyecto del Fondo Global.

El agente comunitario debe ser de la localidad.

Los Combi se deben realizar en localidades con altos índices de malaria.

* Los Combi no se deben realizar en zonas con alteración del orden público.

* En el momento en el que se prevean picos de malaria deben hacerse campañas. 
Correspondencia entre la política de control y las dinámicas socioecológicas de la malaria

La figura 7 integra la información obtenida relacionando los componentes de la PCM con procesos socioecológicos. Puede observarse que los procesos de la política de control solo dan respuesta a las dinámicas de primer orden. El análisis sugiere que si bien la PCM realiza acciones como diagnóstico, reporte y notificación de casos, compra y distribución de toldillos, control de calidad a las placas y entrega de medicamentos, estas acciones no tienen el alcance suficiente para intervenir, influir o contrarrestar el efecto que tienen los procesos sociales y ecológicos que están relacionados con la presencia de la malaria en la localidad como la minería, el conflicto armado y el fenómeno del niño.

\section{Discusión}

En el ámbito del análisis de políticas públicas en salud, el interés por el tema de la sostenibilidad de las políticas es reciente y de escasa exploración. Aunque se reconoce la necesidad de comprender cómo las crisis económicas y sociales pueden afectar las políticas, o cómo pueden reconfigurarse para sobreponerse a las crisis (13), el tema ha sido poco estudiado. El modelo utilizado en esta investigación tampoco había sido aplicado antes a una política sanitaria.

Las publicaciones científicas que analizan la sostenibilidad de una política pública en salud son escasas. Una búsqueda realizada hasta el 9 de octubre del 2013 en las bases de datos de PubMed y Science Direct, utilizando los campos de búsqueda de título y abstract, sin restricciones de tiempo y combinando los siguientes términos: Sustainability $A N D$ public health policies, Sustainability $A N D$ health policies, Sustainability AND health policy, arrojó 331 artículos, de los cuales solo siete correspondían a investigaciones centradas en aspectos de orden institucional que, a pesar de las crisis, hicieran posible la permanencia de las políticas o el logro de los beneficios en salud que estas pretendían. Los artículos restantes se pueden agrupar en cuatro grandes categorías: impactos ambientales de políticas públicas, efectos sobre la salud humana de determinadas políticas públicas,

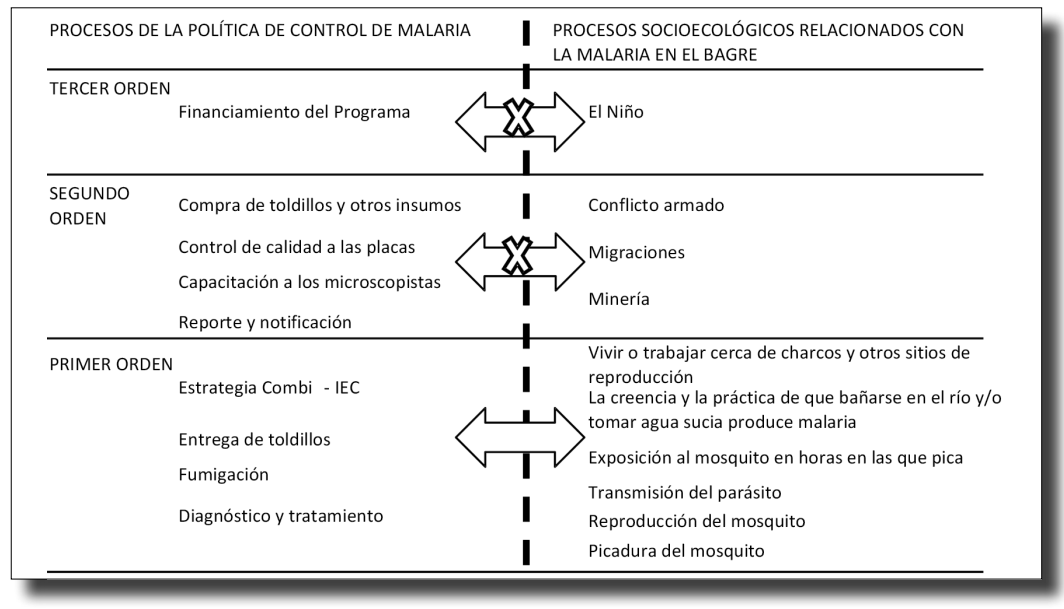

Figura 7. Correspondencia ENTRe PRocesos de LA PCM y PROCESOS SOCIOECOLÓgicos Relacionados CON LA MALARIA. SíNTESIS DE LOS DATOS OBTENIDOS

Fuente: elaboración propia 
sostenibilidad de programas o intervenciones en salud pública, y aspectos financieros en programas de salud pública o de los sistemas de salud. Ninguno de estos artículos exploró las características de diseño institucional que condicionan la sostenibilidad de políticas públicas. Posiblemente, el trabajo más próximo a este propósito es el de Gruen et ál. (14), quienes hacen una revisión de estudios sobre la sostenibilidad de diferentes programas de salud y construyen un modelo. No obstante, este modelo es insuficiente para apoyar la interpretación de nuestros hallazgos, debido a que su escala de observación es más pequeña y concreta (programas específicos) y sus resultados y conclusiones no son siempre extrapolables a políticas públicas más complejas; en segundo lugar, no incluye el análisis de las relaciones de poder, una categoría esencial en las políticas públicas $\mathrm{y}$, finalmente, no precisa la definición de sostenibilidad que sustenta el modelo.

En el ámbito de la investigación en sostenibilidad, el concepto de resiliencia, particularmente la resiliencia socioecológica, ha dado lugar a un enfoque para entender la sostenibilidad de sistemas socioecológicos $(15,16)$. Esta perspectiva, que se ha desarrollado en mayor medida a través del estudio de sistemas de gestión de bienes comunales y de recursos naturales, sugiere que los sistemas tienden a tener capacidad adaptativa a perturbaciones cuando sus arreglos instituciones dan lugar a organizaciones policéntricas (1720), tienen diversidad (21-23), mecanismos de realimentación $(19,24)$ y eficiencia $(21)$.

A pesar de que las políticas públicas de control de malaria no son sistemas de gestión de bienes comunales, sino de bienes públicos, la utilización del modelo es apropiada. Estas políticas son sistemas de gestión de bienes públicos debido a que los beneficios en salud generados por el control de la malaria son de disfrute conjunto de la población y no es posible la exclusión de beneficiarios. En este tipo de sistemas se ha demostrado que algunas características de diseño institucional de sistemas sostenibles de gestión de recursos de uso, son similares a algunos sistemas eficientes de gestión de bienes públicos como los servicios de policía y las economías locales de áreas metropolitanas urbanas. Características como las estructuras policéntricas, los mecanismos de monitoreo, la diversidad y la eficiencia se encuentran entre tales semejanzas (25).

En el contexto de las políticas de control de malaria, las perturbaciones del sistema corresponden a dinámicas socioecológicas relacionadas con la malaria y su control. En esta investigación se pudo identificar que la minería, las variaciones climáticas, el conflicto armado, la migración de personas y las prácticas socioculturales son procesos relacionados con la dinámica y el control de la malaria en el municipio analizado. Estas dinámicas no necesariamente son las mismas en todas las localidades donde hay malaria ni en todas las épocas. De hecho, la política mundial de erradicación de la malaria de mediados del siglo xx fracasó, en parte, debido a la implementación de modelos de intervención estándar que no reconocían la variabilidad de las condiciones locales (26, pp. 45-7) y a una planeación que no se adaptaba a éstas (26, pp. 63-5).

La relación entre malaria y minería también ha sido descrita en países como Ghana y Brasil. En zonas donde hay explotaciones mineras, esta enfermedad se agudiza en lugares donde existen métodos precarios de extracción de oro, deficiente infraestructura en las viviendas y deficientes condiciones ocupacionales de aquellas personas que trabajan en actividades relacionadas con la minería (27-29). 
La minería también favorece la migración poblacional y con ello se hace más difícil el control de la malaria. Las zonas de extracción minera atraen nuevos asentamientos humanos. El aumento de población en estas zonas favorece a su vez la movilidad de personas infectadas y la difusión de la enfermedad a zonas con baja incidencia (27). A diferencia de las poblaciones establecidas localmente, las poblaciones migrantes presentan una mayor incidencia de malaria; el mayor riesgo se ha asociado con deficientes condiciones de vida, asentamientos improvisados con viviendas construidas en materiales de pobre calidad, ubicación cerca de áreas pantanosas, y falta de medios para comprar mosquiteros $(30,31)$.

La policentricidad se considera una característica esencial de los sistemas sostenibles porque permite a los sistemas de actores configurar formas de gobierno ajustadas a la escala de los problemas que deben afrontar $(25,32)$, genera diversidad en tanto los agrupamientos más locales son diferentes entre sí, y ello a su vez permite que los sistemas de actores resuelvan más rápidamente la amplia variedad de problemas que puedan afrontar en el nivel local. Cuando hay formas de organización policéntrica, los sistemas de actores tienen más unidades productoras de servicios en el nivel local que bajo formas centralizadas, los conflictos se resuelven más rápido, porque hay en una instancia cercana, y se genera diversidad, porque la autonomía que existe en diferentes jurisdicciones muestra diferentes alternativas para resolver un problema (25). En el sistema de actores identificado en El Bagre hay un actor central - la SDS - que controla los recursos y presta los servicios directamente a los beneficiarios en las localidades; los demás actores, como las secretarías locales de salud, han venido perdiendo autonomía y relevancia en la provisión de servicios; adicionalmente, la interacción con la SDs ha generado una distancia entre los beneficiarios y el proveedor de servicios, lo que hace difícil detectar y resolver oportunamente los conflictos. En términos generales, este sistema de actores no tiene una configuración policéntrica, tiene escasa diversidad, pocos mecanismos de realimentación, y a pesar de su efectividad demostrada durante el año 2011, no es clara su eficiencia a mediano y largo plazo. En estas condiciones, el sistema denota una limitada capacidad para adaptar las reglas y dispositivos de control a las dinámicas socioecológicas y, por consiguiente, la PCM podría considerarse poco sostenible, al menos desde la óptica del modelo empleado aquí.

Desde la perspectiva teórica, la presencia de actores centrales dentro de las estructuras que gobiernan los sistemas socioecológicos no debe asociarse mecánicamente con un efecto adverso para el desempeño del sistema, los sistemas suelen requerir la existencia de actores centrales; particularmente en localidades distantes, donde el costo de salida es alto para los locales, y se pueden formar "tiranías locales" (33, p. 282). Por su parte, las estructuras multinivel y descentralizadas tampoco pueden asumirse directamente como diseños institucionales policéntricos. El estudio realizado por Lieberman (34) para establecer el impacto de los modelos de gobierno sobre el control de las enfermedades infecciosas, encontró que las estructuras policéntricas están asociadas a limbos en las responsabilidades de las autoridades y limitaciones en la prestación efectiva de los servicios. Sin embargo, Lieberman asumió la policentricidad como descentralización y no analizó las reglas en uso de los sistemas de actores que daban lugar a tales estructuras descentralizadas. El gobierno policéntrico de un sistema se identifica a través de las reglas en uso del sistema de actores y no a través de la identificación del número de actores por responsabilidad; en tal sentido, la modularidad o policentricidad y su contribución a la 
sostenibilidad del sistema no constituyen un asunto cuantitativo, sino uno cualitativo (35).

El tipo de organización institucional centralizada explica, en parte, la escasa diversidad encontrada en el sistema de actores. Así, las acciones de control de la malaria en las localidades responden a directrices del órgano central y no a unidades ubicadas en las localidades. Ello limita las posibilidades de generar cursos alternativos de acción porque las acciones implementadas en las localidades obedecen al mismo actor, lo que a su vez reduce las posibilidades de aprendizaje e innovación en las decisiones y acciones de los actores, al momento de responder a los cambiantes dinámicas socioecológicas locales y regionales relacionadas con la malaria.

Las reglas descritas de este sistema de actores también revelaron escasos mecanismos de realimentación. La notificación de casos y las auditorías a los microscopistas son un ejemplo de algunos mecanismos. Sin embargo, no hay mecanismos que permitan a los actores reconocer el comportamiento de las dinámicas sociales y ecológicas de la malaria. Carecer de dicho mecanismo dificulta ajustar la escala de gobierno a la escala del problema, y reduce las posibilidades de llevar a cabo acciones de control que se adapten a esas características locales para ser efectivas. Lo anterior explica, parcialmente, por qué las reglas de acción de la PCM en El Bagre no se corresponden con las dinámicas socioecológicas de la malaria en dicha localidad.

La política de control de malaria que se está llevando a cabo en el municipio ha mostrado ser efectiva, en tanto los casos de malaria en el 2011 se redujeron. Esta disminución está relacionada, en parte, con la implementación del PFG. Aunque el programa regular de control de malaria en Antioquia ya venía 144 fortaleciéndose, dicho proyecto incrementó los recursos financieros, toldillos, pruebas rápidas y dispositivos móviles para el reporte en línea de casos, mejoró el manejo de información y vinculó más personal, incluyendo auditores de microscopistas y profesionales de diferentes áreas.

En este contexto, mantener la efectividad observada en los últimos años parece difícil, si se tienen en cuenta, además, los costos sociales y monetarios que la sustentan. Para el 2015 se prevé la finalización del PFG, lo que supone una reducción de recursos; por otra parte, el pago a los microscopistas y personas de la comunidad que hizo el PFG aumentó momentáneamente la cobertura, pero afectó los intereses de los líderes comunitarios y su motivación para participar de manera voluntaria en estas actividades.

\section{Conclusión}

A pesar de que la PCM analizada ha mostrado un cierto grado de efectividad en los últimos años, las posibilidades de mantener la malaria controlada en el largo plazo podrían ser reducidas. Esto se debe, principalmente, a tres razones. Primero, se prevé una disminución de recursos que sustentan las labores que se venían haciendo durante el 2011, particularmente cuando finalice el proyecto del Fondo Global en el 2015. Segundo, los procesos socioecológicos como el fenómeno del Niño, la minería y las migraciones, que influyen en el comportamiento de la malaria en la localidad, no son tenidos en cuenta por las reglas o acciones del sistema de actores, no son objeto de la PCM y tampoco parece haber incentivos para que esta realidad cambie. En tercer lugar, el sistema de actores presenta una escasa capacidad de adaptar sus reglas y modos de acción a las dinámicas socioecológicas de la malaria. La complejidad de este tipo de políticas hace necesario realizar más estudios similares de casos, que contribuyan a la comprensión de su sostenibilidad. 


\section{Agradecimientos}

A la Universidad de Antioquia por el apoyo financiero, a la Estrategia de Sostenibilidad de la Vicerrectoría de Investigación, Universidad de Antioquia, 2013-2014 para el Grupo de Epidemiología, a la Gobernación de Antioquia, al profesor Daniel Ruiz Carrascal, y a Carol Barajas de la Fundación Ideas para la Paz por el suministro de datos.

\section{Referencias bibliográficas}

1. Gómez-Arias R. Gestión de políticas públicas. Aspectos operativos. Rev Fac Nac Salud Pública. 2012; 30 (2): 223-36.

2. Walt G, Shiffman J, Schneider H, Murray S, Brugha R, Gilson L. 'Doing' Health Policy Analysis: Methodological and Conceptual Reflections and Challenges. Health Policy and Planning. 2008; 23 (5): 308-17.

3. Salas-Zapata W. Diseño de un modelo de análisis de sostenibilidad de políticas públicas en salud. Barcelona: Universidad Politécnica de Cataluña; 2012.

4. Jiménez M, Hinestroza Y, Gómez R. Reformas sanitarias e impacto del control de malaria en dos áreas endémicas de Colombia. Colombia Médica. 2007; 38 (2): 113-31.

5. Carmona-Fonseca J. La malaria en Colombia, Antioquia y las zonas de Urabá y Bajo Cauca: panorama para interpretar la falla terapéutica antimalárica. Iatreia. 2003; 16 (4): 299-18.

6. Pattanayak S, Dickinson K, Corey C, Murray B, Sills E, Kramer R. Deforestation, Malaria, and Poverty: A Call for Transdisciplinary Research to Support the Design of Cross-Sectoral Policies. Sustainability: Science, Practice \& Policy. 2006; 2 (2): 45-56.

7. Poveda G, Rojas W. Evidencias de la asociación entre brotes epidémicos y el fenómeno de El Niño-Oscilación del Sur. Revista de la Academia Colombiana de Ciencias Exactas, Físicas y Naturales. 1997; 21 (81): 421-9.

8. Departamento Administrativo Nacional de Estadística (DANE). Boletín Censo 2005 [Internet] [acceso: 23 de feberero del 2012].

9. Vargas J, Arias G, Arcila L. Perfil de la región del Bajo Cauca. Medellín: Dirección de Planeación Estratégica Integral; 2009.

10. Secretaría Seccional de Salud y Protección Social de Antioquia (sssa). Eventos en salud pública. Enfermedades transmitidas por vectores 2000-2010 por municipio. Medellín: sssa; 2013.

11. Vasquez L, Rodríguez A, Calvo Y, Benavides A. Los saberes y las prácticas de la población sobre la malaria y su influencia en la efectividad de los tres puestos de diagnóstico y tratamiento: Municipio de El Bagre, Antioquia, Colombia, 1994. Medellín: Universidad de Antioquia; 1996.
12. Ruiz D, Poveda G, Vélez I, Quiñones M, Rúa G, Velásquez L et ál. Modelling Entomological-Climatic Interactions of Plasmodium Falciparum Malaria Transmission in Two Colombian Endemic-Regions: Contributions to a National Malaria Early Warning System. Malaria Journal. 2006; 5 (66): 1-30.

13. Ricciardi W, Corrao G. Walking across Evidence and Sustainability of Public Health Policies - Do we Need a New Journal? Italian Journal of Public Health. 2012; 9 (3): 1-2.

14. Gruen R, Elliot J, Nolan M, Lawton P, Parkhill A, McLaren C et ál. Sustainability Science: An Integrated Approach for Health-Programme Planning. Lancet. 2008; 372 (1): 1579-89.

15. Folke C. Resilience: The Emergence of a Perspective for Social-Ecological Systems. Global Environ Chang. 2006; 16 (3): 253-67.

16. Boyd E, Folke C. Adapting Institutions. Governance, Complexity and Social-Ecological Resilience. Cambridge, UK: Cambridge University Press; 2012.

17. Low B, Ostrom E, Simon C, Wilson J. Redundancy and Diversity: Do They Influence Optimal Management? En: Berkes F, Colding J, Folke C, editores. Navigating Social-Ecological Systems: Building Resilience for Complexity and Change. Cambridge: Cambridge University Press; 2003. pp. 83-114.

18. Norberg J, Cumming G. Networks. En: Norberg J, Cumming G, editores. Complexity Theory for a Sustainable Future. Nueva York: Columbia University Press; 2008. pp. 81-4.

19. Ostrom E. El gobierno de los bienes comunes. La evolución de las instituciones de acción colectiva, segunda edición. México D. F: Universidad Nacional Autónoma de México; 2011.

20. Webb C, Bodin Ö. A Network Perspective on Modularity and Control of Low in Robust Systems. En: Norberg J, Cumming G, editores. Complexity Theory for a Sustainable Future. Nueva York: Columbia University Press; 2008. pp. 86-118.

21. Fiksel J. Designing Resilient, Sustainable Systems. Environ Sci Technol. 2003; 37: 5330-9.

22. Holling C. Understanding the Complexity of Economic, Ecological, and Social Systems. Ecosystems. 2001; 4: 390-405.

23. Norberg J, Wilson J, Walker B, Ostrom E. Diversity and Resilience in Social-Ecological Systems. En: Norberg J, Cumming G, editores. Complexity Theory for a Sustainable Future. Nueva York: Columbia University Press; 2008. pp. 46-80.

24. Norberg J, Cumming G. Information Processing. En: Norberg J, Cumming G, editores. Complexity Theory for a Sustainable Future. Nueva York: Columbia University Press; 2008. pp. 149-54.

25. Ostrom E. Polycentric Systems as One Approach for Solving Collective Action Problems. Working Papers; 2008.

26. Nájera JA. Malaria Control: Achievements, Problems and Strategies. Parassitologia. 2001; 43 (1-2): 1-89.

27. Barbieri A, Sawyer D. Heterogeneity of Malaria Prevalence in Alluvial Gold Mining Areas in Northern Mato Grosso State, Brazil. Cadernos de Saúde Pública. 2007; 23 (12). 
28. Barbieri A, Sawyer D, Soares-Filho B. Population and Land Use Effects on Malaria Prevalence in the Southern Brazilian Amazon. Human Ecology. 2005; 33: 847-74.

29. Asante K, Zandoh C, Dery D, Brown C, Adjei G, Antwi-Dadzie Y et ál. Malaria Epidemiology in the Ahafo Area of Ghana. Malaria Journal. 2011; 10 (211): 1-14.

30. Mitjà O, Paru R, Selve B, Betuela I, Siba P, De Lazzari E et ál. Malaria Epidemiology in Lihir Island, Papua New Guinea. Malaria Journal. 2013; 12 (1): 1-8.

31. Teklehaimanot A, Mejía P. Malaria and Poverty. Annals Of The New York Academy Of Sciences. 2008; 1136: 32-7.
32. McGinnis M, Ostrom E. Reflections on Vincent Ostrom, Public Administration and Policentricity. Public Administration Review. 2011; 72 (1): 15-25.

33. Ostrom E. Understanding Institutional Diversity. Princeton: Princeton University Press; 2005.

34. Lieberman E. The Perils of Polycentric Governance of Infectious Disease in South Africa. Social Science \& Medicine. 2011; 73: 676-84.

35. Janssen M, Bodin O, Anderies J, Elmqvist T, Ernstson H, McAllister R et ál. A Network Perspective on the Resilience Of Social-Ecological Systems. Ecology and Society. 2006; 11 (1): 1-15. 